\title{
Imaging of intramuscular haemangiomas of the extremities
}

Nuttaya Pattamapaspong ${ }^{1}$, MD, Wilfred CG $\underline{P e h}^{2}$, FRCP, FRCR, Tony WH $\underline{S h e k}^{3}$, MBBS, FRCPA

\begin{abstract}
Intramuscular haemangiomas are benign soft tissue tumours that are more frequently seen in children and young adults. As they may be difficult to diagnose clinically, imaging has an important role in the detection, diagnosis and preoperative planning of these lesions. Haemangiomas of the extremities may be classified into capillary, cavernous, venous and mixed types, with or without an arteriovenous shunt, depending on the predominant vascular channels. Nonvascular components such as fat, smooth muscle, fibrous tissue and thrombus may also be present. This pictorial essay highlights the imaging features of intramuscular haemangiomas, with an emphasis on magnetic resonance imaging.
\end{abstract}

Keywords: arteriovenous haemangioma, cavernous haemangioma, intramuscular haemangioma, vascular malformation, venous haemangioma

\section{INTRODUCTION}

Intramuscular haemangiomas are benign soft tissue tumours that are more frequently encountered in children and young adults. Their clinical features are variable, as they may remain undetected for years, or may present as a painless mass or a mass with vague pain after exercise. In the absence of skin discolouration, physical findings in intramuscular haemangiomas are often nonspecific. Therefore, imaging, particularly magnetic resonance (MR) imaging, has an important role in detection, diagnosis and preoperative planning of these lesions. In this pictorial essay, we present the imaging features of haemangiomas, emphasising the characteristic features of intramuscular haemangiomas on MR imaging.

\section{CLASSIFICATION}

Haemangiomas represent a broad spectrum of benign neoplasms that histologically resemble blood vessels. Haemangiomas are pathologically classified into four types based on the predominant type of vascular channel identified within the lesion; capillary, cavernous, venous and arteriovenous (AV) haemangiomas. ${ }^{(1,2)}$ However, some lesions are known as mixed-type haemangiomas because they have an admixture of different types of vessels. Apart from vascular channels, nonvascular components may be present, particularly in the cavernous type of haemangioma (Fig. 1). The nonvascular components include fat, smooth muscle, fibrous tissue, thrombus, calcification and bone. ${ }^{(2)}$

\section{CLINICAL PRESENTATION}

The clinical presentation of intramuscular haemangiomas is non-specific. Patients usually present with chronic pain and a new palpable mass. Pain is often worsened with exercise due to vascular dilatation and increased regional blood flow, causing swelling and compressive pain. (3) These lesions may intermittently increase in size when placed in a dependent position or during straining, but this change may be difficult to detect in deep-seated lesions. Skin discolouration may be present if the lesion involves the skin and subcutaneous tissue. ${ }^{(2)}$

\section{GENERAL IMAGING FEATURES}

Imaging features reflect the vascular and nonvascular components of haemangiomas. Although haemangiomas are usually either not visible or seen as a non-specific soft tissue mass, radiographs play a major role in detection of calcifications and bone changes. Phleboliths, which are characteristic calcifications present in cavernous and venous haemangiomas, are seen in $20 \%$ of intramuscular haemangiomas ${ }^{(4)}$ (Fig. 2). Amorphous or curvilinear calcifications may be seen. ${ }^{(2)}$ Regional bone changes related to haemangiomas include periosteal reaction (Fig. 2), cortical erosion (Figs. $3 \& 4$ a), reactive sclerosis (Fig. 4a), coarsening of the trabeculae (Fig. 2) and bony overgrowth. ${ }^{(4)}$

On unenhanced computed tomography $(\mathrm{CT})$, haemangiomas are seen as poorly defined lesions of similar attenuation as

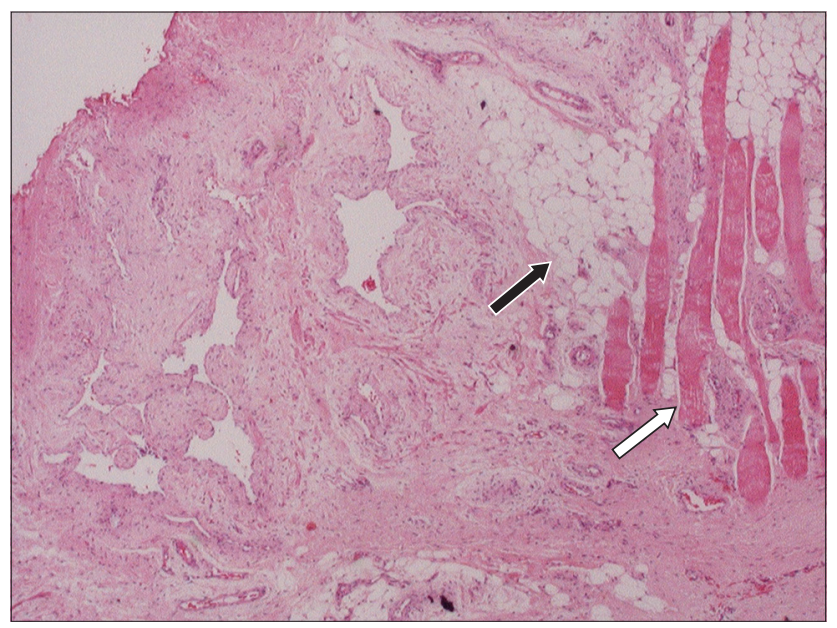

Fig. 1 Photomicrograph shows an intramuscular haemangioma that is composed of well-formed vessels as well as some mature fat (black arrow) adjacent to the skeletal muscle fibres (white arrow) (Haematoxylin \& eosin, $\times 10)$.

${ }^{1}$ Department of Radiology, Chiang Mai University, Chiang Mai, Thailand, ${ }^{2}$ Department of Diagnostic Radiology, Khoo Teck Puat Hospital, Singapore, ${ }^{3}$ Department of Pathology, The University of Hong Kong, Queen Mary Hospital, Hong Kong SAR

Correspondence: Prof Wilfred CG Peh, Clinical Professor and Head, Department of Diagnostic Radiology, Khoo Teck Puat Hospital, 90 Yishun Central, Singapore 768828. wilfred.peh@ktph.com.sg 


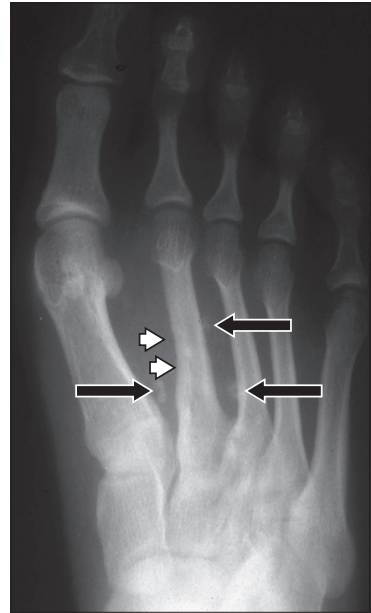

Fig. 2 Radiograph of the foot of a patient with a cavernous haemangioma shows multiple phleboliths (arrows) and an adjacent periosteal reaction in the second metatarsal bone (arrowheads).

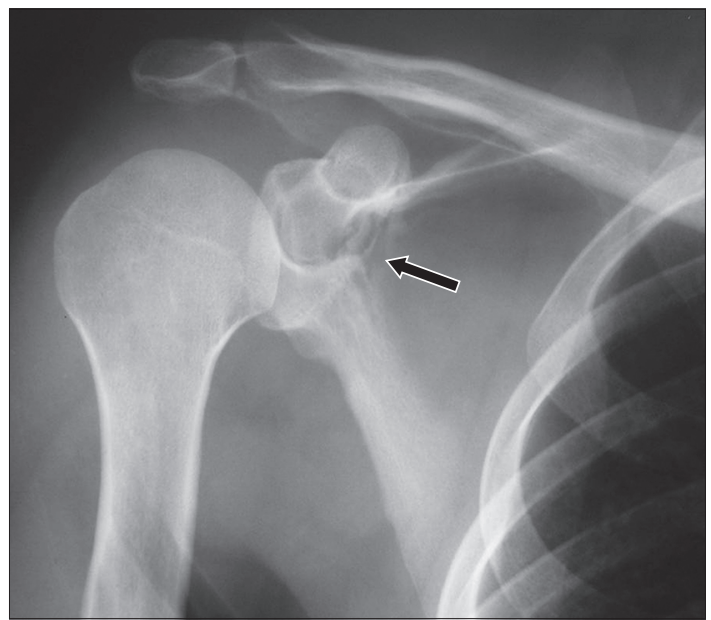

Fig. 3 Radiograph of the shoulder of a patient with cavernous haemangioma shows erosion in areas of the scapula near the glenoid fossa (arrow), with areas of coarsened trabeculae.

skeletal muscle. After intravenous contrast administration, haemangiomas have a variable degree of enhancement. ${ }^{(2)}$ Small haemangiomas may have intense enhancement similar to that of the adjacent blood vessels. Large haemangiomas may have intense enhancement of the peripheral area and serpentine vascular component, or may have no enhancement. ${ }^{(5,6)}$ Area of fat overgrowth may be detected. Phleboliths are best seen on CT and their detection leads to a specific diagnosis (Fig. 5). Although $\mathrm{CT}$ is able to detect haemangiomas, MR imaging is far superior to CT in demonstrating the vascular component and full extent of the lesion. ${ }^{(7)}$ On ultrasonography (US), a haemangioma is seen as a complex mass with vascular channels. Phleboliths can also be detected if they are large enough. US is useful to evaluate superficial haemangiomas (Fig. 6). However, for deep-seated lesions, US may be unable to identify the entire extent of the lesion, thus limiting its accuracy in the evaluation of haemangiomas. ${ }^{(9)}$

On MR imaging, both the vascular and nonvascular components of haemangiomas are depicted. The vascular channels are seen as a tangle of tubular structures with hypointense to isointense signal on T1-weighted images and
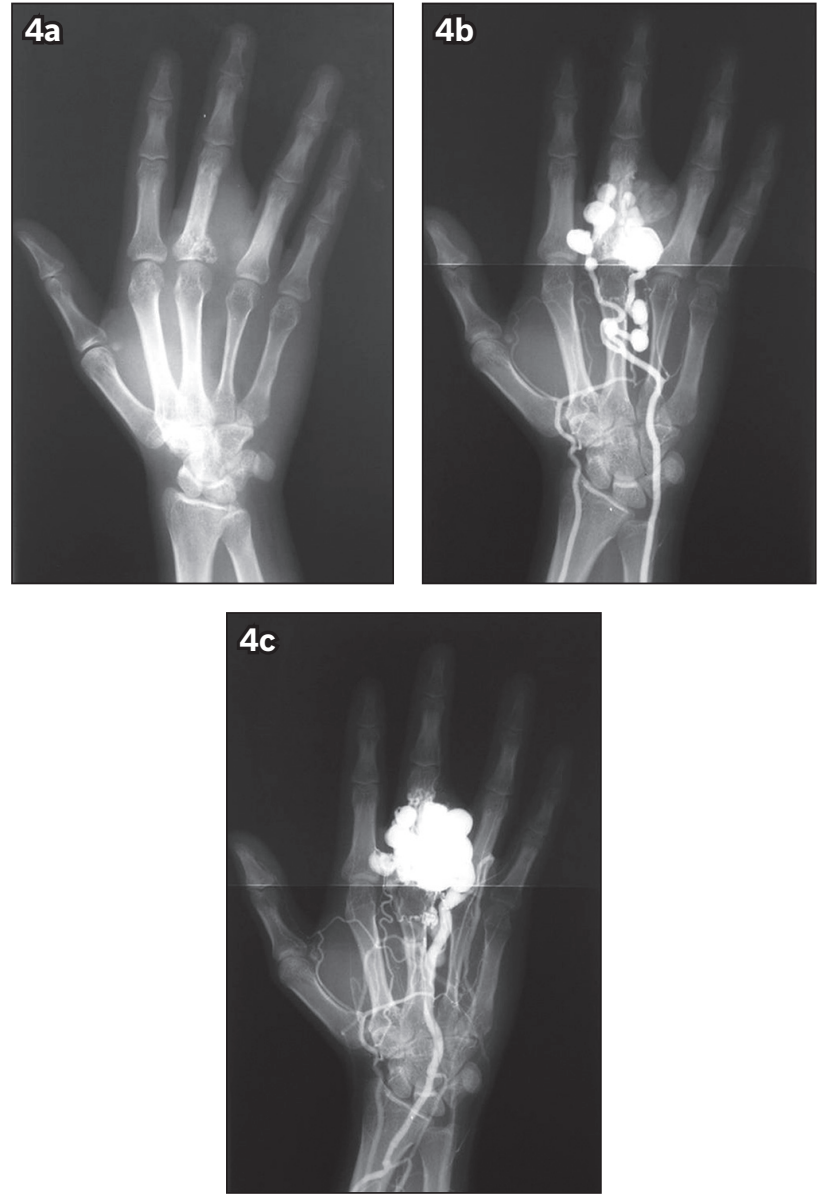

Fig. 4 (a) Radiograph of a patient with arteriovenous haemangioma of the hand shows a soft tissue mass with bony erosion and reactive sclerosis affecting the proximal phalanx of the middle finger. ( $b$ \& $c$ ) Angiograms show markedly dilated and tortuous feeding arteries and prominent arteriovenous shunting.

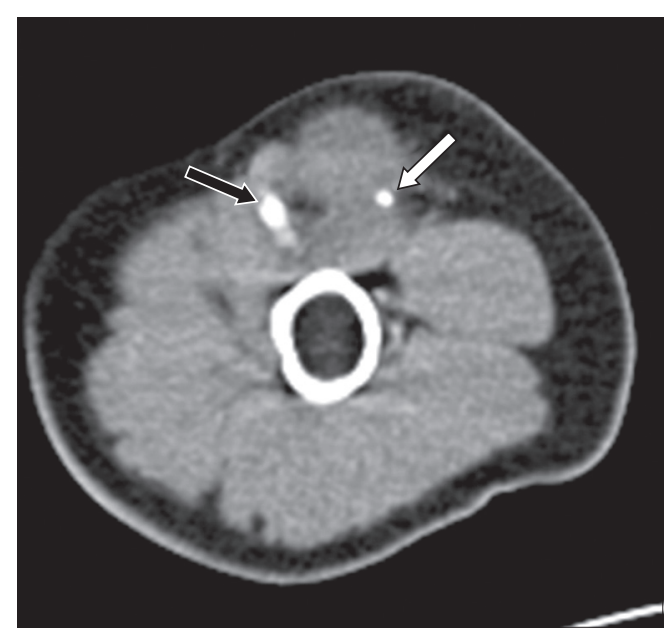

Fig. 5 Axial post-contrast CT image of cavernous haemangioma shows a phlebolith (white arrow) within an ill-defined, poorly enhancing mass located in the biceps brachii muscle. The brachial artery and adjacent brachial vein are enhanced (black arrow).

hyperintense signal on T2-weighted images relative to muscle. ${ }^{(2)}$ The flow voids seen on all pulse sequences indicate rapid blood flow. ${ }^{(10)}$ On T1-weighted images, fat overgrowth is seen as areas of hyperintensity. Phleboliths appear as circular areas of hypointensity. Haemorrhage may be detected as areas of 

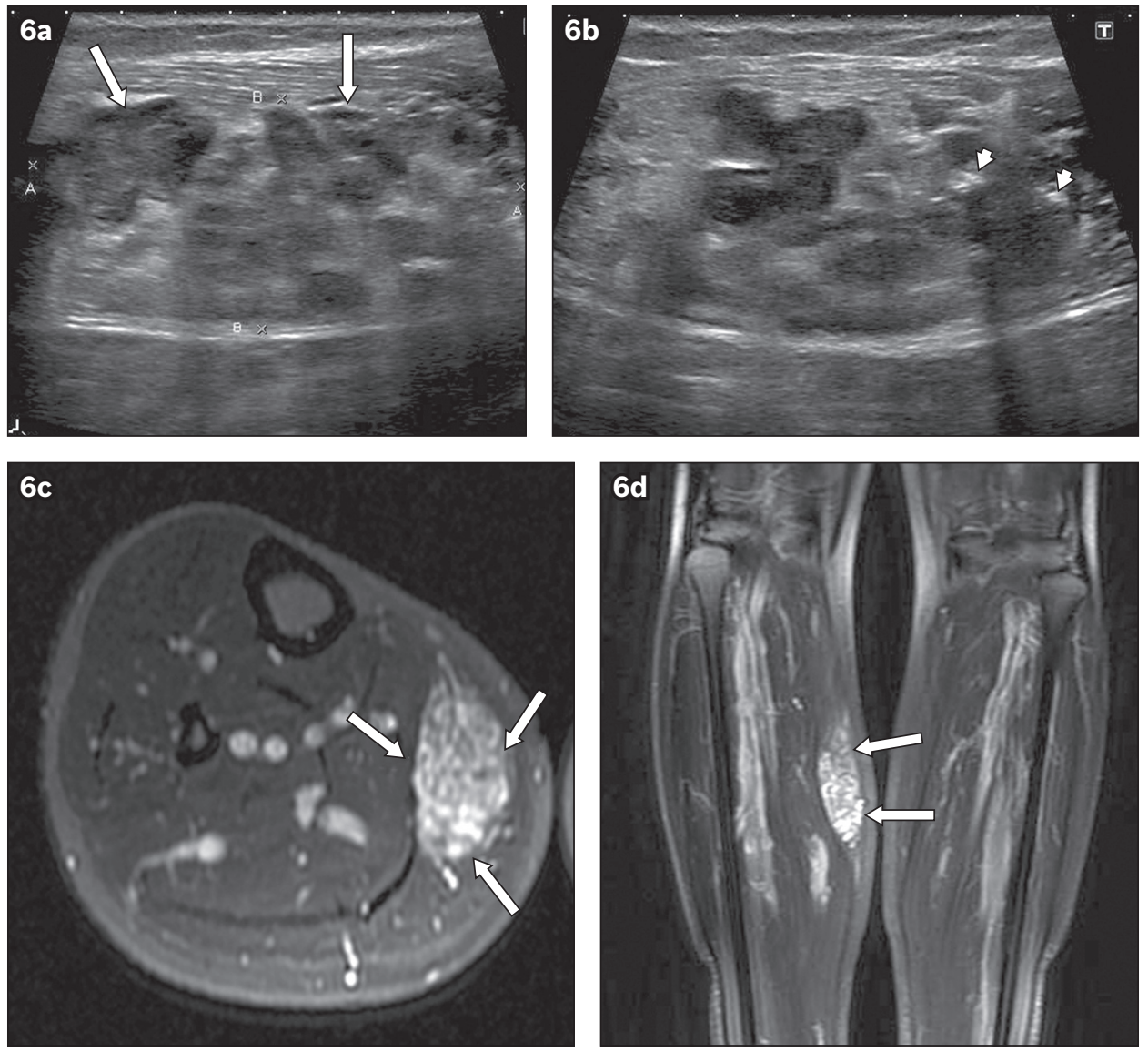

Fig. 6 Superficially located cavernous haemangioma in a 29-year-old man. (a \& b) Longitudinal US images show an ill-defined mass with prominent vascular channels (arrows) and phleboliths within (arrowheads). Corresponding (c) axial and (d) coronal T2-weighted MR images show typical features of a well-marginated, predominantly markedly hyperintense, heterogeneous mass within the medial gastrocnemius muscle (arrows)
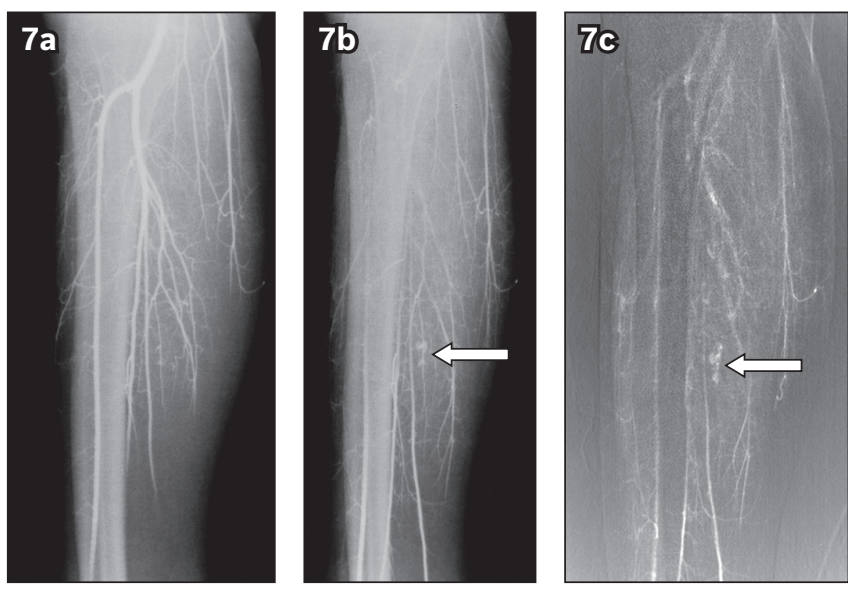

Fig. 7 Nonsubtracted serial angiograms of a calf venous haemangioma taken at (a) five seconds and (b) ten seconds and (c) a digital subtraction image taken at 20 seconds show normal arteries and gradual filling of a small tangle of blood vessels (arrows).

hyperintense signal on T1-weighted images, with fluid-fluid levels on T2-weighted images. ${ }^{(11)}$ On angiography, variable findings are associated with haemangiomas, depending on the types of vascular components (Figs. 4 \& 7). Because MR imaging can evaluate vascular components in a noninvasive fashion, diagnosis of haemangiomas usually does not require angiography.

In clinical practice, MR imaging is the single most useful modality for diagnosis of haemangiomas, because it can characterise the flow rate of the vascular channel and identify the type of nonvascular component. ${ }^{(8)}$ Radiographs complement MR imaging in detecting calcifications. Haemangiomas may be detected by US or CT, but the advantages of US and CT are fewer than those of MR imaging. Angiography is reserved for further imaging of AV haemangiomas to identify the feeding arteries and draining veins, usually prior to embolisation. The MR imaging protocol for extremity lesions should include the axial and longitudinal planes - with the longitudinal plane being either a coronal or sagittal plane depending on lesion location. The pulse sequences typically comprise T1- and T2-weighted sequences and post-contrast enhanced T1-weighted images. The latter are obtained with application of fat suppression for better lesion delineation.

\section{IMAGING FEATURES IN SPECIFIC TYPES OF HAEMANGIOMAS \\ Capillary haemangioma}

Capillary haemangiomas are composed of small vessels lined by a flattened endothelium. This type of haemangioma usually involves the skin and subcutaneous tissue, and has a typical clinical appearance (Fig. 8a). The majority of capillary haemangiomas spontaneously involute within the childhood period. ${ }^{(2)}$ Although capillary haemangiomas are the most common type, imaging is seldom required for diagnosis. On MR imaging, a capillary haemangioma may be seen as an infiltrative lesion that has an isointense signal on T1-weighted images and iso-to-hyperintense 

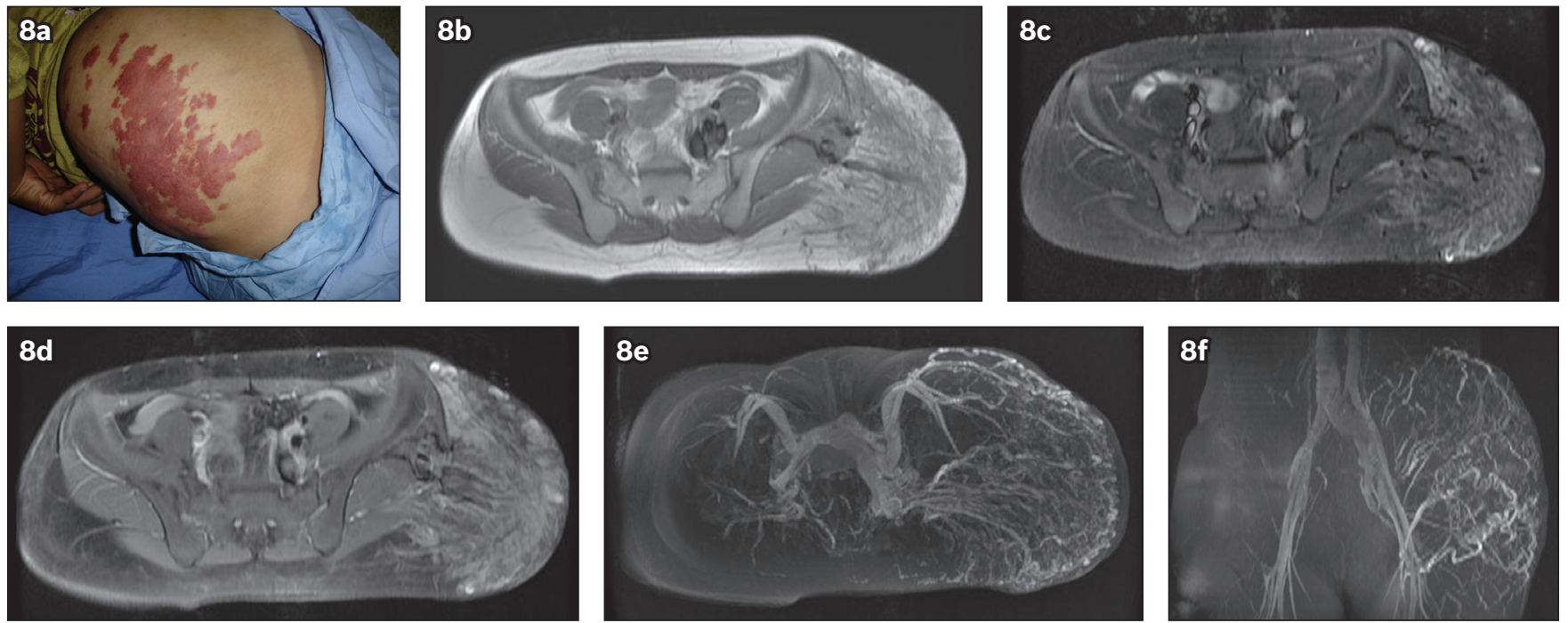

Fig. 8 Longstanding subcutaneous capillary haemangioma with intramuscular involvement in a 20-year-old woman. (a) Clinical photograph of the left buttock shows soft, raised, reddish lesions that are non-tender to the touch. Axial (b) T1-weighted, (c) T2-weighted and (d) post-contrast T1-weighted MR images show an extensive infiltrative T1-isointense and T2-hyperintense lesion that is intensely enhancing. Although it is mostly located in the subcutaneous fat plane, there is involvement of the underlying gluteus muscles. (e) Axial and (f) coronal two-dimensional time-of- flight MR angiography images show the feeding arteries from the left internal and external iliac arteries. The left common iliac vein is enlarged due to the increase in blood flow from the draining veins.
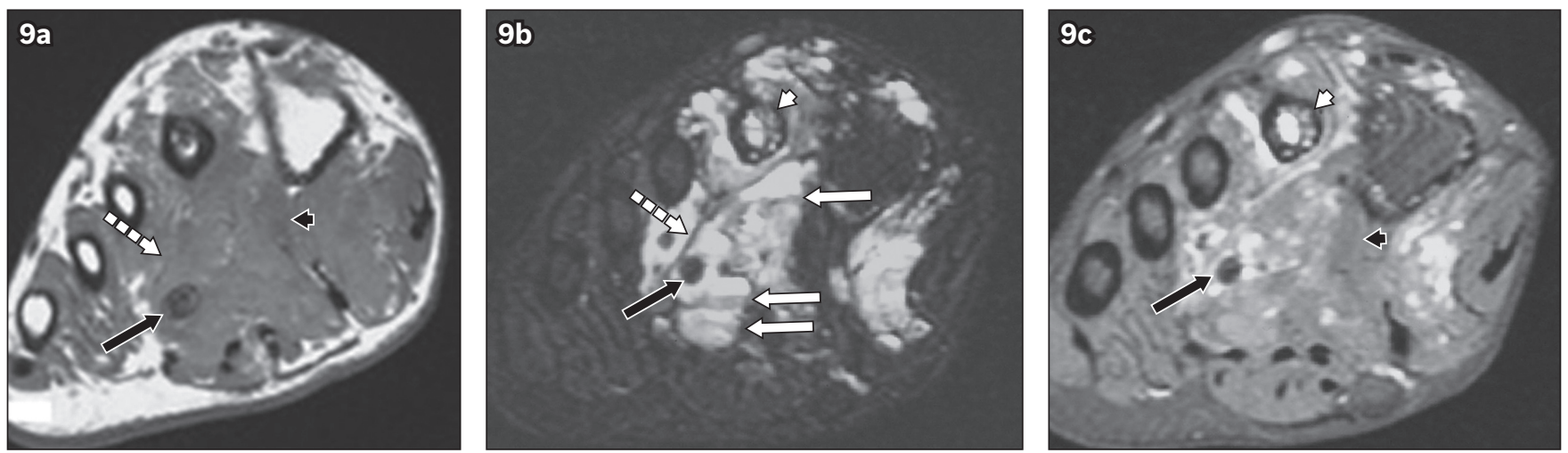

Fig. 9 Intramuscular cavernous haemangioma of the foot with subcutaneous and bone involvement. Axial (a) T1-weighted, (b) T2-weighted and (c) postcontrast T1-weighted MR images show T1-isointense and T2-hyperintense signal relative to muscle within an extensively infiltrating lesion. Contrast enhancement is mild and heterogeneous. Fluid-fluid levels due to previous bleeding are seen (white arrows). Non-enhancing components are due to phleboliths (black arrows), fibrous septa (dashed arrows) and smooth muscles (black arrowheads). Note the vascular channels in the cortical bone and medullary cavity of the metatarsal bone (white arrowheads).

signal on T2-weighted images relative to muscle (Figs. 8b-c). The lesion is highly vascular and enhances intensely after intravenous contrast administration $^{(12)}$ (Figs. 8d-f). Unlike the other types of haemangiomas, the vascular channels are usually too small to appreciate, and fat overgrowth is unusual. ${ }^{(2)}$ Compared to those in superficial locations, intramuscular capillary haemangiomas are rare, usually persist and do not involute. Although imaging shows a heterogeneous intramuscular mass with fast flow and intralesional fat, the need for a biopsy to rule out sarcomas cannot be obviated. ${ }^{(13)}$

\section{Cavernous haemangioma}

Cavernous haemangiomas are composed of dilated blood-filled spaces lined by flattened endothelium. Fat components have been found in as many as $93 \%-100 \%$ of lesions, and are considered a reactive phenomenon rather than a true neoplasm. ${ }^{(2,5)}$ Because the mass does not involute and often requires surgical excision, cavernous haemangioma is the type of haemangioma that most frequently requires imaging for either diagnosis or surgical planning. ${ }^{(7)}$ Phleboliths are commonly seen in cavernous haemangiomas as a result of dystrophic calcification within organising thrombi, and typically appear as rounded T1- and T2-hypointense areas on MR imaging. Amorphous or curvilinear calcification may occur. Pathologically, the amorphous and curvilinear calcifications reflect the mature bone interspersed with large cavernous vascular channels. ${ }^{(14}$

On MR imaging, cavernous haemangiomas appear as heterogeneous masses due to nonvascular components, which consist of fat, fibrous septa, smooth muscle or blood products (Fig. 9). On T1-weighted images, a cavernous haemangioma is seen as a poorly marginated mass of usually isointense, sometimes mildly hyperintense, signal (Fig. 10a). Areas of markedly high signal intensity on T1-weighted images represent fat components, which are also often present. ${ }^{(2,10)}$ On T2-weighted MR images, 

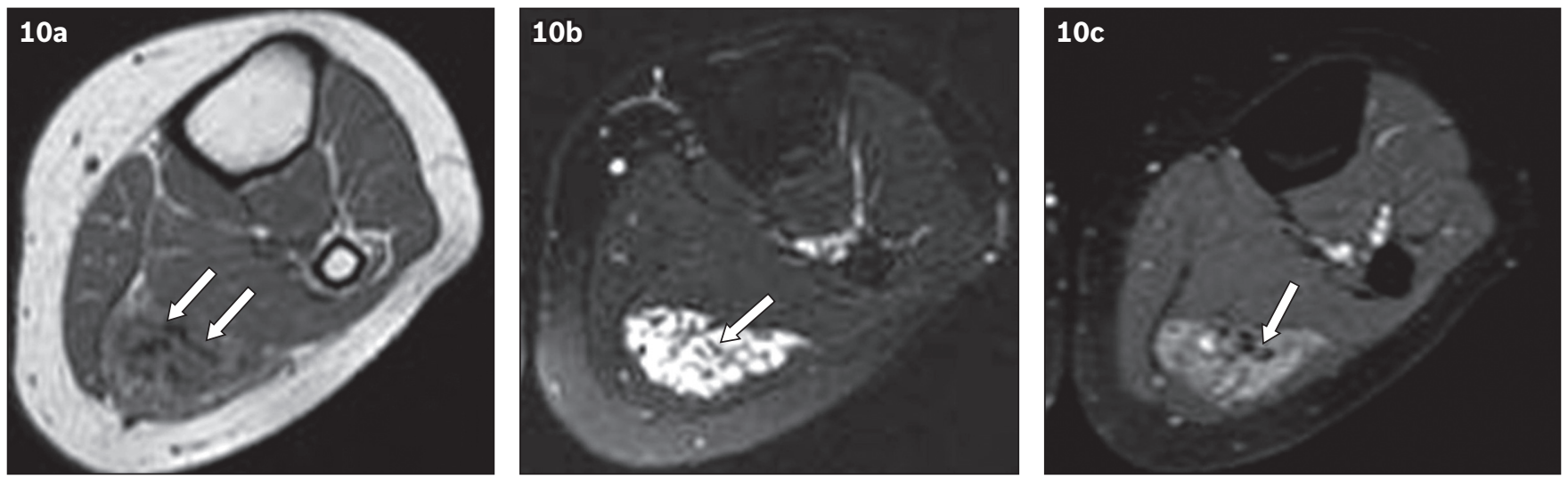

Fig. 10 Intramuscular cavernous haemangioma of the calf. Axial (a) T1-weighted, (b) T2-weighted and (c) post-contrast T1-weighted MR images show T1-isointense and markedly T2-hyperintense signal within a lobulated mass that is well-defined on T2-weighted imaging. Multiple septa are present (arrows). Contrast enhancement is heterogeneous.
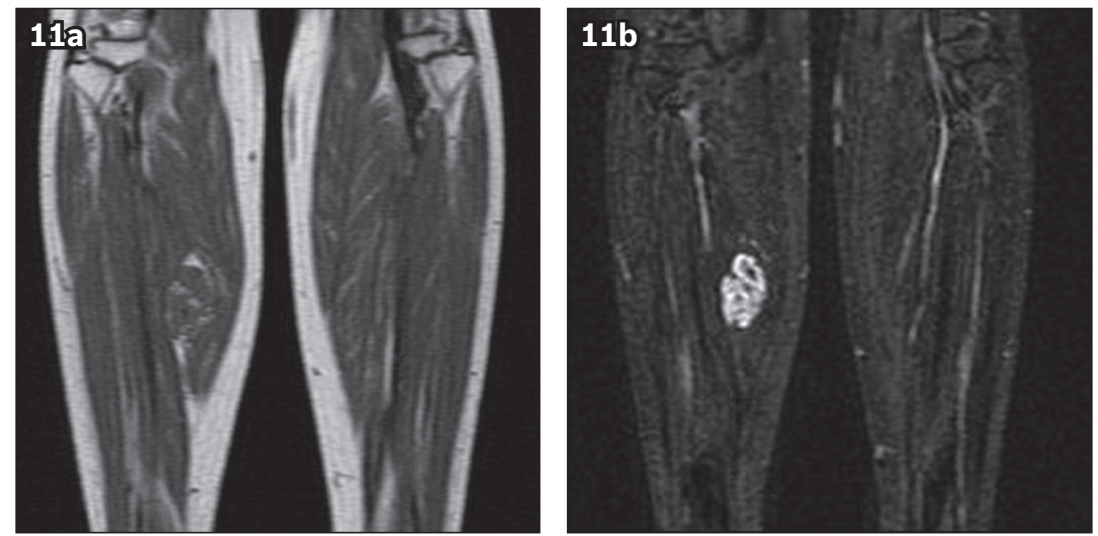

Fig. 11 Intramuscular venous haemangioma of the right calf. Coronal (a) T1-weighted and (b) T2-weighted MR images show T1-isointense and marked T2hyperintense signal within a lesion that has a well-defined serpentine configuration on T2-weighted imaging. Small areas of intralesional fat appear T1-hyperintense.
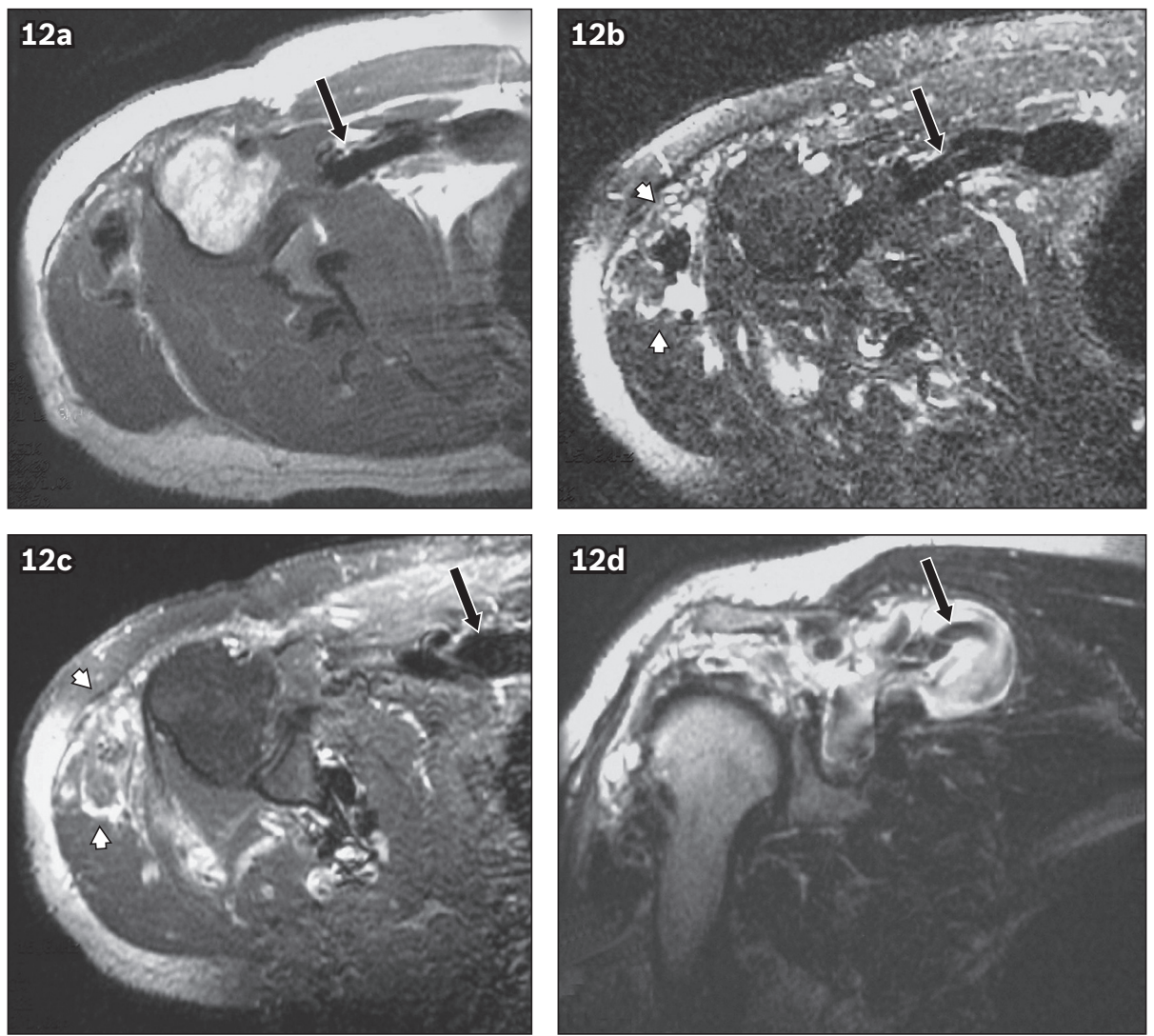

Fig. 12 Mixed arteriovenous and cavernous haemangioma of the shoulder of the patient in Fig. 3. Axial (a) T1-weighted, (b) T2-weighted; and (c) post-contrast T1-weighted and (d) coronal T2-weighted MR images show flow voids in the large vascular channels on all pulse sequences, due to the fast-flow phenomenon (arrows). The rest of the lesion represents the cavernous component, which shows patchy T2-hyperintense areas that are markedly enhanced (arrowheads). 
haemangioma is seen as a well-marginated mass with areas of very high signal intensity due to the cystic vascular spaces (Figs. 6c-d, 9b \& 10b). The marked enhancement of the mass on post-contrast imaging also reflects the vascular components (Figs. 9c and 10c). On angiography, cavernous haemangiomas show enlarged feeding arteries with AV shunting and contrast puddling. ${ }^{(15)}$

\section{Venous haemangioma}

Venous haemangiomas are predominantly composed of vessels with thick muscular walls. These haemangiomas have slow blood flow. Thrombus and phleboliths may be found within these lesions. ${ }^{(2)}$ On MR imaging, the serpentine vascular channels are seen as areas of T1-isointensity and marked T2-hyperintensity relative to muscles (Fig. 11). Although venous haemangiomas may show contrast puddling on delayed images, no abnormality is usually present on angiography ${ }^{(2)}$ (Fig. 7).

\section{Arteriovenous haemangioma}

AV haemangiomas are composed of abnormal communications between arteries and veins. This type of haemangioma is characterised by high blood flow due to the AV shunting. Occasionally, stenosis and thrombus may cause reduced flow and mimic a mass. ${ }^{(16)}$ Clinical abnormalities associated with high-flow lesions include venous distention, bruit and increased overlying skin temperature. In patients with a large AV shunt that increases cardiac output, Branham sign, which is a reflex bradycardia after compression of the shunt, may occur. ${ }^{(7)}$ On MR imaging, AV haemangiomas contain high-flow vascular channels, which are seen as curvilinear or serpentine areas of flow void on all pulse sequences. Because they frequently contain areas of capillary or cavernous haemangioma, a combination of features may occur, and such AV haemangiomas are sometimes called mixed-type haemangiomas (Fig. 12). Angiography of AV haemangiomas shows markedly tortuous feeding vessels with intense tumour staining and early draining veins (Figs. 4b-c).

\section{CONCLUSION}

Intramuscular cavernous, venous and AV haemangiomas have typical features on MR imaging. As clinical findings are usually non-specific, recognition of imaging appearances aids in detection and arriving at a confident diagnosis. Accurate diagnosis by MR imaging helps to avoid unnecessary surgery. In cases where surgery is required, MR imaging aids in preoperative planning.

\section{REFERENCES}

1. Fletcher CDM, Bridge JA, Hogendoorn PCW, Mertens F, eds. WHO Classification of Tumours of Soft Tissue and Bone. 4th ed. Lyon: IARC Press, 2013.

2. Kransdorf MJ, Murphey MD. Vascular and lymphatic tumors. In: Kransdorf MJ, Murphey MD, eds. Imaging of soft tissue tumors. 3rd ed. Philadelphia, PA: Lippincott Williams \& Wilkins, 2014: 179-230.

3. Wierzbicki JM, Henderson JH, Scarborough MT, et al. Intramuscular hemangiomas. Sports Health 2013; 5:448-54.

4. Sung MS, Kang HS, Lee HG. Regional bone changes in deep soft tissue hemangiomas: radiographic and MR features. Skeletal Radiol 1998; 27:205-10.

5. Griffin N, Khan N, Thomas JM, Fisher C, Moskovic EC. The radiological manifestations of intramuscular haemangiomas in adults: magnetic resonance imaging, computed tomography and ultrasound appearances. Skeletal Radiol 2007; 36:1051-9.

6. Levine E, Wetzel LH, Neff JR. MR imaging and CT of extrahepatic cavernous hemangiomas. AJR Am J Roentgenol 1986; 147:1299-304.

7. Murphey MD, Fairbairn KJ, Parman LM, et al. From the archives of the AFIP. Musculoskeletal angiomatous lesions: radiologic-pathologic correlation. Radiographics 1995; 15:893-917.

8. Buetow PC, Kransdorf MJ, Moser RP Jr, Jelinek JS, Berrey BH. Radiologic appearance of intramuscular hemangioma with emphasis on MR imaging. AJR Am J Roentgenol 1990; 154:563-7.

9. Ahuja AT, Richards P, Wong KT, Yuen EH, King AD. Accuracy of high-resolution sonography compared with magnetic resonance imaging in the diagnosis of head and neck venous vascular malformations. Clin Radiol 2003; 58:869-75.

10. Rak KM, Yakes WF, Ray RL, et al. MR imaging of symptomatic peripheral vascular malformations. AJR Am J Roentgenol 1992; 159:107-12.

11. Tsai JC, Dalinka MK, Fallon MD, Zlatkin MB, Kressel HY. Fluid-fluid level: a nonspecific finding in tumors of bone and soft tissue. Radiology 1990; 175:779-82.

12. Vilanova JC, Barceló J, Smirniotopoulos JG, et al. Hemangioma from head to toe: MR imaging with pathologic correlation. Radiographics 2004; 24:367-85.

13. Yilmaz S, Kozakewich HP, Alomari Al, et al. Intramuscular capillary-type hemangioma: radiologic-pathologic correlation. Pediatr Radiol 2014; 44: 558-65.

14. Nagira K, Yamamoto T, Marui T, et al. Ossified intramuscular hemangioma: multimodality imaging findings. Clin Imaging 2001; 25:368-72.

15. Levin DC, Gordon DH, McSweeney J. Arteriography of peripheral hemangiomas. Radiology 1976; 121(3 Pt 1):625-30.

16. Pulidori M, Capuano C, Mouchaty H, Cioffi F, Di Lorenzo N. Intramuscular thrombosed arteriovenous hemangioma of the upper right arm mimicking a neuroma of the ulnar nerve: case report. Neurosurgery 2004; 54:770-2. 


\section{SINGAPORE MEDICAL COUNCIL CATEGORY 3B CME PROGRAMME} (Code SMJ 202003B)

Question 1. Concerning the clinical presentation of intramuscular haemangiomas:

(a) They may present as painless masses.

(b) Pain may be noticeable after exercise.

(c) Skin discolouration is common in cavernous haemangiomas.

(d) Large arteriovenous (AV) haemangiomas may result in increased cardiac output.

Question 2. Regarding imaging of intramuscular haemangiomas:

(a) Radiographs help in detection of bone erosion.

(b) Ultrasonography is the best imaging technique for the detection of phleboliths.

(c) Computed tomography always shows intense lesion enhancement similar to that of adjacent blood vessels.

(d) Angiography is usually reserved for lesions that require embolisation.

Question 3. Nonvascular tissue components that are commonly found in intramuscular haemangiomas include:

(a) Nerve fibres.

(b) Fat.

(c) Smooth muscle.

(d) Calcification.

Question 4. Regarding the magnetic resonance (MR) imaging features of cavernous haemangiomas:

(a) Fluid-fluid levels represent areas of previous bleeding.

(b) Phleboliths are hypointense on T2-weighted images.

(c) The masses usually appear well-circumscribed on T1-weighted images.

(d) Areas of marked T2-hyperintense signal within the lesion indicate vascular channels.

Question 5. Regarding the imaging features of AV haemangiomas:

(a) On MR imaging, AV haemangiomas contain high-flow vascular channels which are seen as curvilinear or serpentine areas of flow void on all pulse sequences.

(b) AV shunting does not occur in association with cavernous haemangioma.

(c) Enlarged tortuous feeding vessels with intense tumour staining and early draining veins are characteristic features.

(d) Angiography plays no useful role.

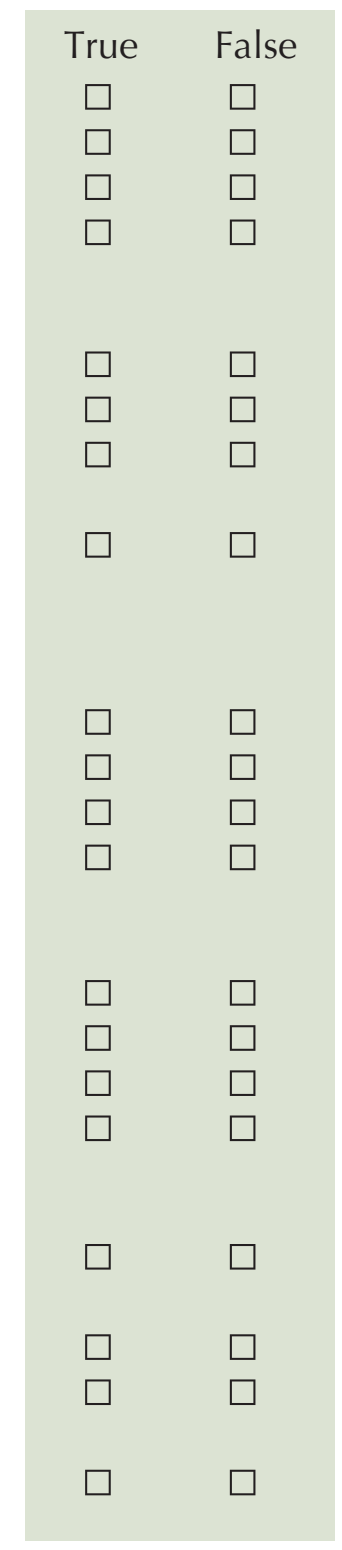

\section{Doctor's particulars:}

Name in full:

Specialty:

MCR no.:

Email:

\section{SUBMISSION INSTRUCTIONS}

Visit the SMJ website: http://www.smj.org.sg/current-issue and select the appropriate quiz. You will be redirected to the SMA login page.

For SMA member: (1) ) Log in with your username and password (if you do not know your password, please click on 'Forgot your password?'). (2) Select your answers for each quiz and click 'Submit'.

For non-SMA member: (1) Create an SMJ CME account, or log in with your SMJ CME username and password (for returning users). (2) Make payment of SGD 21.40 (inclusive of $7 \%$ GST) via PayPal to access this month's quizzes. (3) Select your answers for each quiz and click 'Submit'.

RESULTS:

(1) Answers will be published online in the SMJ May 2020 issue. (2) The MCR numbers of successful candidates will be posted online at the SMJ website by 11 May 2020. (3) Passing mark is $60 \%$. No mark will be deducted for incorrect answers. (4) The SMJ editorial office will submit the list of successful candidates to the Singapore Medical Council. (5) One CME point is awarded for successful candidates. (6) SMC credits CME points according to the month of publication of the CME article (i.e. points awarded for a quiz published in the March 2020 issue will be credited for the month of March 2020, even if the deadline is in May 2020).

Deadline for submission (March 2020 SMJ 3B CME programme): 12 noon, 4 May 2020. 